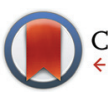

CrossMark \&lick for updates

Cite this: Polym. Chem., 2015, 6 , 3694

Received 30th January 2015, Accepted 31st March 2015

DOI: 10.1039/c5py00141b

www.rsc.org/polymers

\title{
High molecular weight mechanochromic spiropyran main chain copolymers via reproducible microwave-assisted Suzuki polycondensation $\uparrow$
}

\author{
Lukas Metzler, ${ }^{a}$ Thomas Reichenbach, ${ }^{b}$ Oliver Brügner, ${ }^{b}$ Hartmut Komber, ${ }^{c}$ \\ Florian Lombeck, ${ }^{a}$ Stefan Müllers, ${ }^{a}$ Ralf Hanselmann, ${ }^{a}$ Harald Hillebrecht, ${ }^{d}$ \\ Michael Walter ${ }^{b, e, f}$ and Michael Sommer ${ }^{* a, b, f}$
}

\begin{abstract}
Suzuki-Miyaura polycondensation (SPC) is widely used to prepare a variety of copolymers for a broad range of applications. Although SPC protocols are often used in many instances, the limits of this method and issues of molecular weight reproducibility are not often looked at in detail. By using a spiropyranbased (SP) mechanochromic copolymer, we present an optimized protocol for the microwave-assisted synthesis of a mechanochromic, alternating copolymer P(SP-alt- $\left.\mathrm{C}_{10}\right)$ via SPC that allows the reproduction of molecular weight distributions. Several parameters such as microwave power, temperature, stoichiometry, and ligand are screened, leading to molecular weights up to $M_{w} \sim 174 \mathrm{~kg} \mathrm{~mol}^{-1}$. The process of optimization is guided by NMR end group analysis which shows that dehalogenation, oxidative deborylation and SP cleavage are the limiting factors that impede further increase of molar mass, while other classical side reactions such as protiodeborylation are not observed. Embossing films of $\mathrm{P}\left(\mathrm{SP}-\right.$ alt $\left.-\mathrm{C}_{10}\right)$ yields the colored merocyanine $(\mathrm{MC})$ copolymer $\mathrm{P}\left(\mathrm{MC}\right.$-alt- $\left.\mathrm{C}_{10}\right)$ that undergoes a thermally facilitated back reaction to $\mathrm{P}\left(\mathrm{SP}-\right.$ alt $\left.-\mathrm{C}_{10}\right)$. DFT suggests that the barrier of the $\mathrm{SP} \rightarrow \mathrm{MC}$ transition has two contributions, with the first one being related to the color change and the second one to internal bond reorganizations. The barrier height is $1.5 \mathrm{eV}$, which suggests that the ease of the thermally facilitated back reaction is either due to residual energy stored in the deformed polymer matrix, or arises from an MC isomer that is not in the thermodynamically most stable state.
\end{abstract}

\section{Introduction}

Suzuki-Miyaura polycondensation (SPC) was established by Schlüter et al. in the 1990 s and is nowadays a standard method to many functional polymers with outstanding mechanical, gas storage, catalytic, luminescence, or opto-electronic

\footnotetext{
${ }^{a}$ Makromolekulare Chemie, Universität Freiburg, Stefan-Meier-Straße 31, 79104 Freiburg, Germany.E-mail: michael.sommer@makro.uni-freiburg.de

${ }^{b}$ Freiburger Materialforschungszentrum, Stefan-Meier-Straße 21, 79104 Freiburg, Germany

${ }^{c}$ Leibniz Institut für Polymerforschung Dresden e.V., Hohe Straße 6, 01069 Dresden, Germany

${ }^{d}$ Institut für Anorganische und Analytische Chemie, Albertstr. 21, 79104 Freiburg, Germany

${ }^{e}$ Fraunhofer Institut für Werkstoffmechanik, Wöhlerstraße 11, 79108 Freiburg, Germany

${ }^{f}$ Freiburger Institut für Interaktive Materialien und Bioinspirierte Technologien, Georges-Köhler-Allee 105, D-79110 Freiburg, Germany

$\dagger$ CCDC 1034507. For crystallographic data in CIF or other electronic format see DOI: $10.1039 / \mathrm{c} 5$ py00141b
}

properties. ${ }^{1-11}$ However, despite the frequent use of SPC, detailed and mechanistic studies that address the fundamental limits of this method are limited, which can be one factor for moderate molar masses obtained can be limited. ${ }^{12-18}$ Concomitant with relatively short chain lengths is the high content of end groups (EG). Depending on the nature and extent of EGs, a non-negligible influence on the material's properties can be expected. In particular, the EGs of conjugated polymers for use in opto-electronic devices are critical as they pose electronic defects which act as quenchers or traps for excited states and charge carriers. ${ }^{19,20}$ From a mechanistic point-of-view, EGs either represent intact chains or side or termination reactions that allow the limiting factors of polycondensation to be revealed. ${ }^{13,21,22}$ Given the molecular weight (MW) dependence of mechanical and opto-electronic properties of conjugated polymers, the optimization of molar mass guided by EG analysis appears to be a particularly fruitful task..$^{4,23,24}$

It is well-known that polymerizations in general and Suzuki-Miyaura cross-coupling reactions in particular can be accelerated by microwave radiation compared to classical 
thermal reactions. ${ }^{25-32}$ At the same time, improvements in purity, yield and the elimination of side reactions are prevalent, which add to the benefits of microwave-accelerated syntheses. ${ }^{27-30}$ Considering the Carothers equation and extensions thereof, the great advantages of such improvements for polycondensation are obvious. ${ }^{33,34}$ However, although the benefits of microwave-assisted SPC are well-recognized, comprehensive protocols are scarce. ${ }^{3,35-40}$ In order to further establish microwave-assisted protocols for transition metalcatalyzed polycondensations, it is important to develop reliable procedures that allow reproducible control over molar masses and hence the correlation of the materials' properties with reaction parameters.

Spiropyrans are molecular switches which respond to multiple external stimuli. ${ }^{41}$ Due to the many different properties of the spiropyran (SP) and merocyanine (MC) forms, a rich equilibrium exists between the less polar, non-conjugated, and colorless SP form and the polar, conjugated, and colored MC form (and several protonated species). ${ }^{42}$ In addition to the various transformations of SP-based materials, mechanical cleavage of the $\mathrm{C}-\mathrm{O}$ bond of SP was recently demonstrated by Moore et al. in solution and bulk using polymer chains that exhibit a single SP unit (mechanophore) at the mid-point of the chain. ${ }^{43,44}$ Since then, this concept has been extended to a variety of SP-based materials that are able to detect chain stretching by developing color. ${ }^{45-51}$ We have recently used Suzuki-Miyaura polycondensation to prepare alternating SP main chain copolymers with different comonomers. ${ }^{52,53}$ Our motivation to use SPC to prepare linear chains with multiple SP incorporation was based on the advantage of a high and tunable mechanophore density. Thus, color intensity can be adjusted to the needs of the experimental method and sample geometry. Another advantage is the possibility to exploit potential cooperative effects of $\mathrm{SP} \rightarrow \mathrm{MC}$ conversion to enhance or accelerate the responsiveness of the material. Such effects are well-known from SP side chain polymers, but have not yet been demonstrated in linear SP copolymers. ${ }^{54}$ In addition, the copolymerization of SP with an aromatic comonomer introduces mesomeric effects which alter the relative stabilities of SP and MC. ${ }^{53}$ Thus, certain pathways between individual species of the complex SP/MC equilibrium might be selectively addressed to generate stable species with high conversion. ${ }^{53,55,56}$ As the mechanical properties of any polymeric material generally depend on entanglements and molecular weight, the reproduction of high molar masses is important. This is especially relevant for polycondensation, where high molar masses critically depend on conversion, which in turn is a complex function of a multitude of parameters.

In this paper we disclose an optimized protocol for the microwave-assisted SPC of a mechanochromic copolymer with alternating SP units and a phenyl-based comonomer $\left(\mathrm{C}_{10^{-}}\right.$ $\left.[\mathrm{B}(\text { pin })]_{2}\right)$. Reproducible molecular weights of $\mathrm{P}\left(\mathrm{SP}-\right.$ alt $\left.-\mathrm{C}_{10}\right)$ up to weight-average molecular weights up to $M_{\mathrm{w}}=174 \mathrm{~kg} \mathrm{~mol}^{-1}$ are achieved using a stock solution approach and the screening of several instrumental and chemical parameters. Variations in stoichiometry, ligand, concentration and amount of the base, power, time, and temperature were carried out. Our protocol further shows that good reproducibility can be obtained with monomer batch sizes as small as $80 \mathrm{mg}$. Side reactions during SPC are monitored by ${ }^{1} \mathrm{H}$ NMR end group analysis, revealing dehalogenation, SP cleavage and oxidative deborylation as main sources that limit further increase in molar mass. Compared to analogous thermal polycondensations, the polymerization time is reduced from days to hours, the molecular weight is higher, and the color of the SP copolymers is much lighter after hot-pressing. Embossed films of $\mathrm{P}\left(\mathrm{SP}-\right.$ alt $\left._{\text {- }} \mathrm{C}_{10}\right)$ exhibit a green color which rapidly disappears on annealing well below the glass transition temperature $\left(\sim 60^{\circ} \mathrm{C}, T_{\mathrm{g}} 129^{\circ} \mathrm{C}\right)$ despite a relatively high barrier of $1.5 \mathrm{eV}$ for the $\mathrm{MC} \rightarrow \mathrm{SP}$ back reaction and very similar energies of SP and MC. This suggests that either energy is stored during the embossing process which is released rapidly at $60{ }^{\circ} \mathrm{C}$, or that the $\mathrm{MC}$ form is not able to find its most stable state during embossing.

\section{Experimental}

\section{Materials}

All starting materials were purchased from Sigma Aldrich. $\mathrm{SPBr}_{2}$ and $\mathrm{C}_{10}-[\mathrm{B}(\mathrm{pin})]_{2}$ were prepared according to recently published procedures. ${ }^{52,53}$ Single crystals of $\left.\mathrm{C}_{10}-\mathrm{B}(\mathrm{pin})\right]_{2}$ were prepared in toluene at $0.125 \mathrm{~mol} \mathrm{l}^{-1}$ at room temperature and left in the fridge at $4{ }^{\circ} \mathrm{C}$ for ten days, collected, and dried at $50{ }^{\circ} \mathrm{C}$. All monomers, ligands and $\mathrm{Pd}_{2} \mathrm{dba}_{3}$ were stored under ambient conditions.

\section{Instrumentation}

All polymerizations were carried out in a CEM Discover-SP microwave reactor and controlled with the Synergy 1.59 software. To generate conditions as reproducible as possible, all reactions were performed in $10 \mathrm{~mL}$ reaction vessels (a change to larger vials requires optimization again due to the different penetration depth of microwave radiation, hence for larger batches thermal heating might be the better choice) under argon with a Teflon septum cap and identical magnetic stir bars. Molecular weights were measured via SEC on a PSS SECcurity HP1200 G1310A from PSS with three columns of pore sizes of $10^{2}, 10^{3}$, and $10^{4} \AA$ and a guard column (PSS). Calibration was done using polystyrene standards. THF was used as the eluent at room temperature at a flow rate of $1.0 \mathrm{~mL}$ $\min ^{-1} \cdot{ }^{1} \mathrm{H}$ NMR $(500.13 \mathrm{MHz})$ and ${ }^{13} \mathrm{C}(125.77 \mathrm{MHz}) \mathrm{NMR}$ spectra were recorded on a Bruker Avance III 500 spectrometer using a $5 \mathrm{~mm}{ }^{1} \mathrm{H} /{ }^{13} \mathrm{C} /{ }^{19} \mathrm{~F} /{ }^{31} \mathrm{P}$ gradient probe. All spectra were recorded at $30{ }^{\circ} \mathrm{C}$ in $\mathrm{CDCl}_{3}$ as the solvent, and were referenced to the residual solvent peak $\delta\left({ }^{1} \mathrm{H}\right) 7.26 \mathrm{ppm} ; \delta\left({ }^{13} \mathrm{C}\right) 77.0 \mathrm{ppm}$. ${ }^{1} \mathrm{H}-{ }^{1} \mathrm{H}$ and ${ }^{1} \mathrm{H}-{ }^{13} \mathrm{C}$ correlated 2D NMR spectra were recorded to verify the signal assignments using standard pulse sequences included in the Bruker TOPSPIN 2.1 software package. The crystal structure data were collected at $100 \mathrm{~K}$ on a Bruker SMART APEX2 CCD area detector diffractometer while using Mo-K $\alpha$ radiation from a microsource (space group $P \overline{1}$, $Z=4, a=9.7994(3), b=12.6632(3), c=14.5262(4) \AA, \alpha=96.185(1)$, 
$\left.\beta=99.296(2), \gamma=109.868(2)^{\circ}\right)$. Data reduction was performed with SAINT (Madison, Wisconsin, USA, 2013), and scaling of the data and absorption correction was performed by SADABS-2012/1 (G. M. Sheldrick, SADABS 2012/1, University of Göttingen, Germany, 2012). The structure was solved by intrinsic phasing using SHELXT (G. M. Sheldrick, SHELXT, University of Göttingen, Germany, 2013) and refined by fullmatrix least-squares minimization on $F^{2}$ using all reflections with SHELXL (5811 reflections (4733 with $I>2 \sigma(I)$ ), 388 parameters, $\left.R_{1}=0.042, \mathrm{w} R_{2}=0.121\right) .{ }^{57}$ The graphical representations were prepared using the software Diamond $3.2 \mathrm{i}^{58}$ CCDC 1034507 contains the supplementary crystallographic data.

\section{Simulations}

The simulations were performed using density functional theory (DFT) where the exchange-correlation energy was approximated at the generalized gradient-corrected approximation as devised by Perdew et al. ${ }^{59}$ Density and wave-functions were represented on real space grids using the projector augmented wave method devised by Blöchl ${ }^{60}$ as implemented in the GPAW package. ${ }^{61,62}$ A grid spacing of $0.2 \AA$ was used for the wave functions. The structures were relaxed in the gasphase without any symmetry constraints until all forces were found to be below $0.05 \mathrm{eV} \AA^{-1}$. The transition path between SP and $\mathrm{MC}$ was explored using the nudged elastic band method. ${ }^{63}$

\section{General comments to the stock solution approach}

The prerequisite to screen many parameters of a SPC reproducibly is a sufficiently large amount of highly pure monomer if powders are directly weighed into the polymerization vessel. In the case of functional monomers, this is not a realistic scenario as monomer scale-up can be highly expensive and time consuming. In addition, the outcome of a screening study is not certain and hence such huge efforts might not be rewarded with success. To develop a reproducible and easy to handle polymerization procedure using small quantities of monomer, stock solutions of monomers, sources of palladium, and ligands were used here. As will be reported, the most important factors to be controlled are stoichiometry and amount of catalyst. Generally, all stock solutions were prepared in degassed toluene and needed to be used in 1-2 days to avoid crystallization of $\mathrm{C}_{10}-[\mathrm{B}(\mathrm{pin})]_{2}$ and degradation of $\mathrm{Pd}_{2} \mathrm{dba}_{3}$. A high boiling point solvent seems prerequisite to avoid changes in concentration during pipetting. The monomer stock solutions prepared in air were then transferred into the microwave vials via pipette and degassed with argon. Catalyst and ligand were added as stock solutions (prepared in dried Schlenk tubes under argon) subsequently under a back stream of argon. All polymerizations were carried out in a CEM Discover microwave reactor with constant temperature and constant irradiation for indicated times.

\section{General procedure for microwave-assisted SPC}

To a $10 \mathrm{~mL}$ microwave vial $2 \mathrm{~mL}$ of a $2 \mathrm{M} \mathrm{K}_{2} \mathrm{CO}_{3}$ solution in water (20 equiv.) and one droplet of pure Aliquat ${ }^{\circledR} 336$ were added. $1 \mathrm{~mL}$ of each of the monomer stock solutions was added (typical size of a monomer stock solution: $2.435 \mathrm{mmol} /$ $25 \mathrm{ml}$ toluene, i.e. $1093.8 \mathrm{mg} \mathrm{SPBr}_{2}$ and $1408.4 \mathrm{mg} \mathrm{C}_{10^{-}}$ $\left.[\mathrm{B}(\mathrm{pin})]_{2}\right)$. Thus, (1,10-bis(4-(4,4,5,5-tetramethyl-1,3,2-dioxaborolan)-phenoxy)decane, $\mathrm{C}_{10^{-}}[\mathrm{B}(\mathrm{pin})]_{2}: 97.4 \mu \mathrm{mol}, 1.00$ equiv. and 5,6'-bis(brom)-1-ethyl-3,3-dimethylspiro[2H-1-benzopyran-2,2'indol], $\mathrm{SPBr}_{2}$ : 1.00 equiv., $97.4 \mu \mathrm{mol}$ ) were added, the fill height was marked, and the mixture was stirred and purged with argon for 30 minutes. The typical size of the $\mathrm{Pd}_{2} \mathrm{dba}_{3}$ stock solution was $2.0 \mathrm{mg} \mathrm{Pd}_{2} \mathrm{dba}_{3}$ in $1 \mathrm{~mL}$ degassed toluene. From this stock solution, $223 \mu \mathrm{L}(0.50 \mathrm{~mol} \%, 0.487 \mu \mathrm{mol})$ was added. The typical size of the SPHOS stock solution was $2.0 \mathrm{mg}$ SPHOS in $1 \mathrm{~mL}$ degassed toluene. From this stock solution, $398 \mu \mathrm{L}(2 \mathrm{~mol} \%, 1.94 \mu \mathrm{mol})$ was added. The mixture was stirred and purged with argon, which led to evaporation of toluene, until an overall fill height corresponding to $3.4 \mathrm{~mL}$ was achieved. Assuming that only toluene evaporated, the final monomer concentration was $\sim 0.14 \mathrm{M}$. The vessel was then transferred into the microwave reactor. The reaction was carried out at various temperatures, microwave powers, and times, whereby the independent variation of power and temperature was enabled by different cooling rates with air (for example: $90{ }^{\circ} \mathrm{C}, 31 \mathrm{~W}, 2 \mathrm{~h}$ ). The stirring speed "high" was selected; this parameter was not varied but is likely to be important as it determines the interfacial area between the aqueous and organic phase. The mixture was cooled to room temperature, separated from the aqueous phase, precipitated into methanol, filtered, and dried at $50{ }^{\circ} \mathrm{C}$ overnight. All reported molecular weights are taken from as-precipitated samples without any re-precipitation, fractionation, or Soxhlet extraction.

\section{Results and discussion}

\section{Optimization of SPC conditions}

Monomer characterization and purity. The intention of this work was to fully elucidate the benefits of microwave synthesis for SPC, and to optimize a protocol for the preparation of high molar masses of the mechanochromic SP main chain copolymer $\mathrm{P}\left(\mathrm{SP}-\right.$ alt- $\left.\mathrm{C}_{10}\right)$ (Scheme 1$)$.

To maximize monomer purity for polycondensation, single crystals of the boronic ester monomer $\mathrm{C}_{10}-[\mathrm{B}(\mathrm{pin})]_{2}$ were grown from toluene. $\mathrm{C}_{10^{-}}[\mathrm{B}(\mathrm{pin})]_{2}$ shows an interesting solid state structure in which all parts of the molecule (i.e. methyl groups, boron, oxygen, phenyl rings and C-10 alkyl chain segment) segregate into domains of equal atoms (Fig. 1). Single crystal data collected at room temperature show a centrosymmetric triclinic unit cell with a halved volume containing two molecules. All distances correspond to the expected values. The thermal displacement parameters of the atoms of the terminal boronic ester group are enlarged according to a higher thermal motion. While no residual solvent was seen in the single crystal structure, ${ }^{1} \mathrm{H}$ NMR spectroscopy showed residual solvents (ethanol and toluene), and as a result, decreased purities of $>98.1 \%$ for $\mathrm{SPBr}_{2}$ and to $>99.1 \%$ for $\mathrm{C}_{10^{-}}$ $[\mathrm{B}(\mathrm{pin})]_{2}$. Interestingly, prolonged storage of the monomers at 


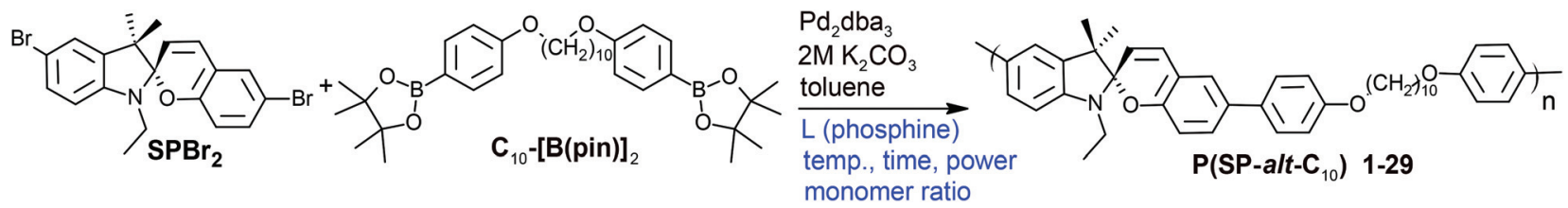

Scheme 1 Suzuki polycondensation of a spiropyran monomer $\left(\mathrm{SPBr}_{2}\right)$ with a flexible comonomer $\left(\mathrm{C}_{10}-\left[\mathrm{B}(\text { pin) }]_{2}\right)\right.$ under various conditions.

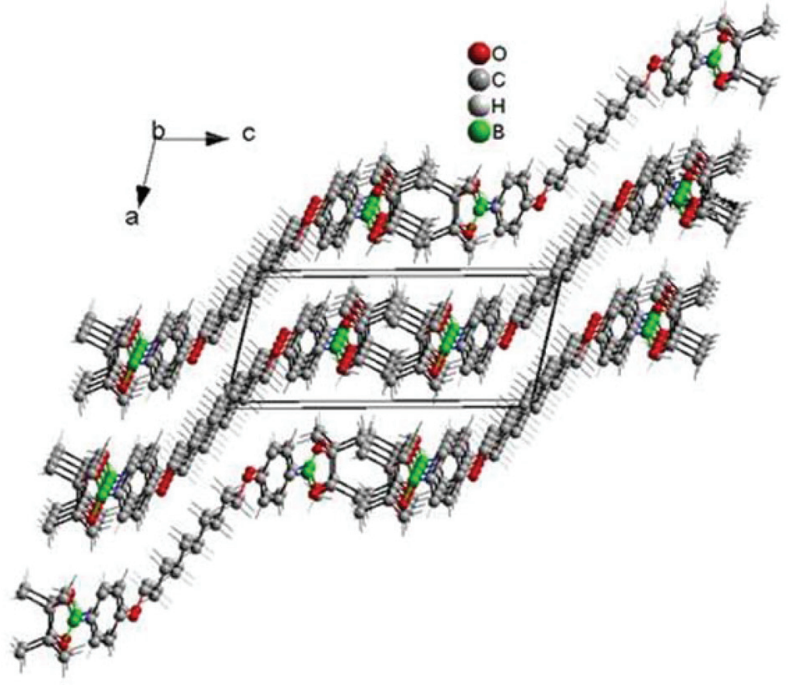

Fig. 1 Unit cell of $\mathrm{C}_{10}-[\mathrm{B}(\mathrm{pin})]_{2}$. All different groups of the molecule (alkyl chain, oxygen, phenyl rings, boron and methyl groups) segregate to form homogeneous domains.

$60{ }^{\circ} \mathrm{C}$ under vacuum did not remove residual solvent. While these values may seem too low for polycondensation, we will show that the presented approach allows for empiric tuning of stoichiometry which is needed to achieve high molar masses, and this way the effect of residual solvent on stoichiometry can be compensated for.

Microwave power and temperature. All SPC entries are compiled in Table 1 . With the aid of stock solutions, small quantities in the range of $\sim 80 \mathrm{mg}$ of $\mathrm{C}_{10^{-}}[\mathrm{B}(\mathrm{pin})]_{2}$ and $\mathrm{SPBr}_{2}$ were reacted under various conditions (Scheme 1). The first polymerization entries were carried out to identify optimum parameters of the microwave reactor. This reactor, especially developed for chemical laboratories, offers many options. Firstly, there is the possibility to adjust temperature, microwave power, speed of stirring and the compressed air cooling separately. Furthermore, the single mode microwave irradiation can be pulsed or irradiated at a constant level until the desired temperature is reached. To obtain highly reproducible results, we chose constant irradiation. To vary power and temperature independently, compressed air cooling was used. To heat and cool at the same time may seem contradictory, but it allows the acceleration of reactions and can suppress undesired side reactions. ${ }^{3,27,31,40}$ Fig. 2 a shows the effect of low and high air cooling, resulting in low and high microwave power
( $3 \mathrm{~W}$ and $25 \mathrm{~W}$ ), respectively, on molecular weight, at a constant temperature of $80^{\circ} \mathrm{C}$ and a reaction time of $2 \mathrm{~h}$. $M_{\mathrm{w}}$ increased from 25 to $47 \mathrm{~kg} \mathrm{~mol}^{-1}$, clearly showing an accelerated polycondensation at higher power (entries 1 and 2, Table 1). Therefore, maximum compressed air cooling enabling maximum microwave power for a given temperature was used throughout, and the temperature was determined by the microwave power only. Importantly, the stirring speed ("high") must be maintained constant, as different stirring velocities lead to different rates of heat dissipation especially at high cooling rates. Next, the reaction temperature, determined by microwave power, was investigated for a constant time of $2 \mathrm{~h}$ (entries 3-8, Table 1, Fig. 2b). This reaction time was chosen on the basis of a compromise between sufficient reaction time and short reaction time to screen several parameters in one day. The reaction temperature and thus microwave power had a large effect on MW, as shown in Fig. 2b. $M_{\mathrm{W}}$ increased from $12.9 \mathrm{~kg} \mathrm{~mol}^{-1}$ at $70{ }^{\circ} \mathrm{C}$ to $50.8 \mathrm{~kg} \mathrm{~mol}^{-1}$ at $90{ }^{\circ} \mathrm{C}$, and then decreased again to $22.9 \mathrm{~kg} \mathrm{~mol}{ }^{-1}$ at $120{ }^{\circ} \mathrm{C}$. As will be explained later in detail using end group analysis, the low molecular weight at $70{ }^{\circ} \mathrm{C}$ arose from incomplete conversion, while the decrease in MW at $T>90{ }^{\circ} \mathrm{C}$ was caused by enhanced side reactions. We will look at these in detail further below, and show that mainly base-induced SP cleavage is responsible for the decrease in MW. At this point we use $90{ }^{\circ} \mathrm{C}$ as the optimum temperature to continue the screening procedure.

Stoichiometry. As determined by the Carothers law, an exact $1: 1$ ratio of bifunctional $\mathrm{AA}$ and $\mathrm{BB}$ monomers is needed to maximize MW. ${ }^{33,64,65}$ Schlüter et al. emphasize that monomer purity and amount is critical for the high MW and reproducibility of a SPC reaction. ${ }^{3,35}$ The stock solution approach is ideal for working with small amounts of monomer, and also to rule out systematic errors and errors from impurities or residual solvents. Thus, the monomer ratio $r=\mathrm{SPBr}_{2}: \mathrm{C}_{10^{-}}$ $[\mathrm{B}(\mathrm{pin})]_{2}$ was easily varied from 0.96 to 1.02 in steps of $1 \%$ (corresponding to variations in volume of $1 \mu \mathrm{L}$ ) (entries 9-14, Fig. 3) without taking the different purities of the two monomers into account.

Somewhat unexpectedly, a slight excess of $\mathrm{C}_{10^{-}}[\mathrm{B}(\mathrm{pin})]_{2}$ of $2 \%$ led to the highest $M_{\mathrm{w}} \sim 110 \mathrm{~kg} \mathrm{~mol}^{-1}$. Due to the slightly lower purity of $\mathrm{SPBr}_{2}$, a value $r>1$ could have been expected to give a maximum $M_{\mathrm{w}}$. However, $M_{\mathrm{w}}$ was doubled for $r=0.98$ compared to $r=1.00$ (Fig. 3). While a similar trend has previously been reported, it is not clear what exactly causes the slight excess of boronic ester to yield higher molar masses. ${ }^{53}$ Contaminants such as oxygen, water, or deborylation were discussed, and Schlüter et al. mentioned that this effect vanishes 
Table 1 Compilation of all SPC reactions. The base was $2 \mathrm{M} \mathrm{K}_{2} \mathrm{CO}_{3}$ in all cases. $M_{\mathrm{w}}$ is used for comparing results throughout, as $M_{n}$ values were affected by the presence of oligomeric species and baseline shifts. The content of oligomers depended on how the polymer precipitated (all polymers were characterized unfractionated, therefore dispersities were large). Data for 9-14 in brackets are from repetition experiments

\begin{tabular}{|c|c|c|c|c|c|c|c|c|c|}
\hline Entry & \multicolumn{2}{|l|}{ Parameter varied } & Power/W & Temp. $/{ }^{\circ} \mathrm{C}$ & Time/min & {$[\mathrm{SPBr} 2]:[\mathrm{BSE}]$} & Ligand & $M_{\mathrm{n}} / \mathrm{kDa}$ & $M_{\mathrm{w}} / \mathrm{kDa}$ \\
\hline 1 & \multirow{2}{*}{\multicolumn{2}{|c|}{$\mu \mathrm{W}$ power }} & 3 & 80 & 120 & 1 & SPhos & 11.6 & 24.8 \\
\hline 2 & & & 25 & 80 & 120 & 1 & SPhos & 17.7 & 46.7 \\
\hline 3 & \multirow{6}{*}{\multicolumn{2}{|c|}{ Reaction temperature }} & 20 & 70 & 120 & 1 & SPhos & 8.4 & 12.9 \\
\hline 4 & & & 25 & 80 & 120 & 1 & SPhos & 20.3 & 47.7 \\
\hline 5 & & & 31 & 90 & 120 & 1 & SPhos & 20.1 & 50.8 \\
\hline 6 & & & 35 & 100 & 120 & 1 & SPhos & 18.6 & 40.5 \\
\hline 7 & & & 43 & 110 & 120 & 1 & SPhos & 17.1 & 36.2 \\
\hline 8 & & & 62 & 120 & 120 & 1 & SPhos & 12.5 & 22.9 \\
\hline 9 & \multirow{7}{*}{\multicolumn{2}{|c|}{ Stoichiometry }} & 31 & 90 & 120 & 0.96 & SPhos & 28.3 & 69.3 \\
\hline 10 & & & 31 & 90 & 120 & 0.97 & SPhos & 22.9 & 53.1 \\
\hline 11 & & & 31 & 90 & 120 & 0.98 & SPhos & $35.6(28.6)$ & $110.3(108.1)$ \\
\hline 12 & & & 31 & 90 & 120 & 0.99 & SPhos & $21.2(16.5)$ & $57.3(39.5)$ \\
\hline 5 & & & 31 & 90 & 120 & 1 & SPhos & $20.1(16.0)$ & $50.8(42.6)$ \\
\hline 13 & & & 31 & 90 & 120 & 1.01 & SPhos & $15.0(20.3)$ & $34.0(59.1)$ \\
\hline 14 & & & 31 & 90 & 120 & 1.02 & SPhos & $18.2(16.7)$ & $42.0(37.1)$ \\
\hline 2 & \multirow[t]{6}{*}{ Reproducibility } & Pd fresh & 25 & 80 & 120 & 1 & SPhos & 17.7 & 46.7 \\
\hline 15 & & Pd old & 25 & 80 & 120 & 1 & SPhos & 16.2 & 33.8 \\
\hline 16 & & m Crystallized & 31 & 90 & 120 & 1 & SPhos & 3.9 & 7.4 \\
\hline 11 & & To be reproduced & 31 & 90 & 120 & 0.98 & SPhos & 35.6 & 110.3 \\
\hline 17 & & Repeat 1 & 31 & 90 & 120 & 0.98 & SPhos & 25.8 & 104.0 \\
\hline 18 & & Repeat 2 & 31 & 90 & 120 & 0.98 & SPhos & 28.6 & 108.0 \\
\hline 17 & \multirow{4}{*}{\multicolumn{2}{|c|}{ Ligand }} & 31 & 90 & 120 & 0.98 & SPhos & 25.8 & 104.0 \\
\hline 19 & & & 31 & 90 & 120 & 0.98 & $\mathrm{P}(o \text {-tolyl })_{3}$ & 12.7 & 25.1 \\
\hline 20 & & & 31 & 90 & 120 & 0.98 & $\mathbf{P}(\text { o-anisyl })_{3}$ & 11.4 & 23.3 \\
\hline 21 & & & 31 & 90 & 120 & 0.98 & $\mathbf{P P h}_{3}$ & 15.5 & 37.1 \\
\hline 22 & \multirow{8}{*}{\multicolumn{2}{|c|}{ Temperature and time }} & 31 & 90 & 10 & 0.98 & SPhos & 11.2 & 21.9 \\
\hline 23 & & & 31 & 90 & 30 & 0.98 & SPhos & 21.9 & 94.1 \\
\hline 24 & & & 31 & 90 & 120 & 0.98 & SPhos & 24.5 & 97.7 \\
\hline 25 & & & 31 & 90 & 180 & 0.98 & SPhos & 21.5 & 77.6 \\
\hline 26 & & & 20 & 70 & 60 & 0.98 & SPhos & 4.7 & 6.7 \\
\hline 27 & & & 20 & 70 & 120 & 0.98 & SPhos & 33.8 & 110.7 \\
\hline 28 & & & 20 & 70 & 180 & 0.98 & SPhos & 26.8 & 157.6 \\
\hline 29 & & & 20 & 70 & 300 & 0.98 & SPhos & 34.8 & 173.9 \\
\hline
\end{tabular}

for gram scale experiments. ${ }^{3}$ However, while the exact reason for the need of an excess of boronic ester remains unknown, the herein used stock solution approach clearly allows the maximization of MW from an empiric point of view. This set of experiments was repeated (except for $r=0.96$ and 0.97), which confirmed $r=0.98$ as the most appropriate monomer ratio in order to maximize MW. The molecular weight obtained for $r=0.96$ did not fit in the row, which may have been caused by invisible crystallization of $\mathrm{C}_{10}[\mathrm{~B}(\mathrm{pin})]_{2}$ at the glass wall of the stock solution from which the monomer was taken, which would have led to a reduction of the concentration of $\mathrm{C}_{10}[\mathrm{~B}(\text { pin })]_{2}$ and a shift of $r$ back towards 0.98 .

Reproducibility. The elucidation of reproducibility of the stock solution approach was one central aim of this study. To this end, the thus far optimized SPC conditions were repeated at different times and using different stock solutions. To our contentment, the developed procedure exhibited good reproducibility (entries 11, 17, 18, Table 1). Fig. 4a shows the SEC curves of three entries made under the same conditions using freshly prepared stock solutions $\left(90^{\circ} \mathrm{C}, 2 \mathrm{~h}, 0.98\right.$ equiv. $\left.\mathrm{SPBr}_{2}\right)$. The SEC curves exhibit an average $M_{\mathrm{w}}=107.4 \pm 2.6 \mathrm{~kg} \mathrm{~mol}^{-1}$ and match very well, and even the intensities in the lower molar mass region are almost identical. $\mathrm{Pd}_{2} \mathrm{dba}_{3}$ stock solu- tions in toluene that were older than one day changed color, which led to decreased MWs (Fig. $4 \mathrm{~b}) . \mathrm{C}_{10}-[\mathrm{B}(\mathrm{pin})]_{2}$ solutions that were older than 1-2 days also did not lead to reproducibility, as the monomer crystallized (with small amounts of crystals at the glass wall being hardly visible) (Fig. 4c). The fact that the curves of Fig. 4a match in all regions almost exactly shows the good reproducibility of this protocol. As a result, new stock solutions were always tested against that result before proceeding with further parameter optimization, and the reproduction of the SEC curves in Fig. 4a was taken as an indicator whether a stock solution was still usable or not.

Ligand and base screening. Having optimized the cooling rate, reaction temperature and monomer ratio, we investigated the influence of the ligand on molecular weight (entries 17, 19-21). The thus far used highly active ligand SPHOS was checked against less expensive alternatives $\mathrm{P}(o \text {-anisyl })_{3}, \mathrm{P}(o$ tolyl $)_{3}$ and $\mathrm{PPh}_{3} \cdot{ }^{17,66}$ However, none of the ligands investigated leads to higher MWs than SPHOS (Fig. 5). At this point the question arises as to what extent reaction parameters are mutually dependent; it might have very well been the case that a $M_{\mathrm{w}}=110 \mathrm{~kg} \mathrm{~mol}^{-1}$ could be obtained for another, cheaper ligand, if it was used from the beginning within a different pathway of parameter optimization..$^{15}$ However, as the permu- 

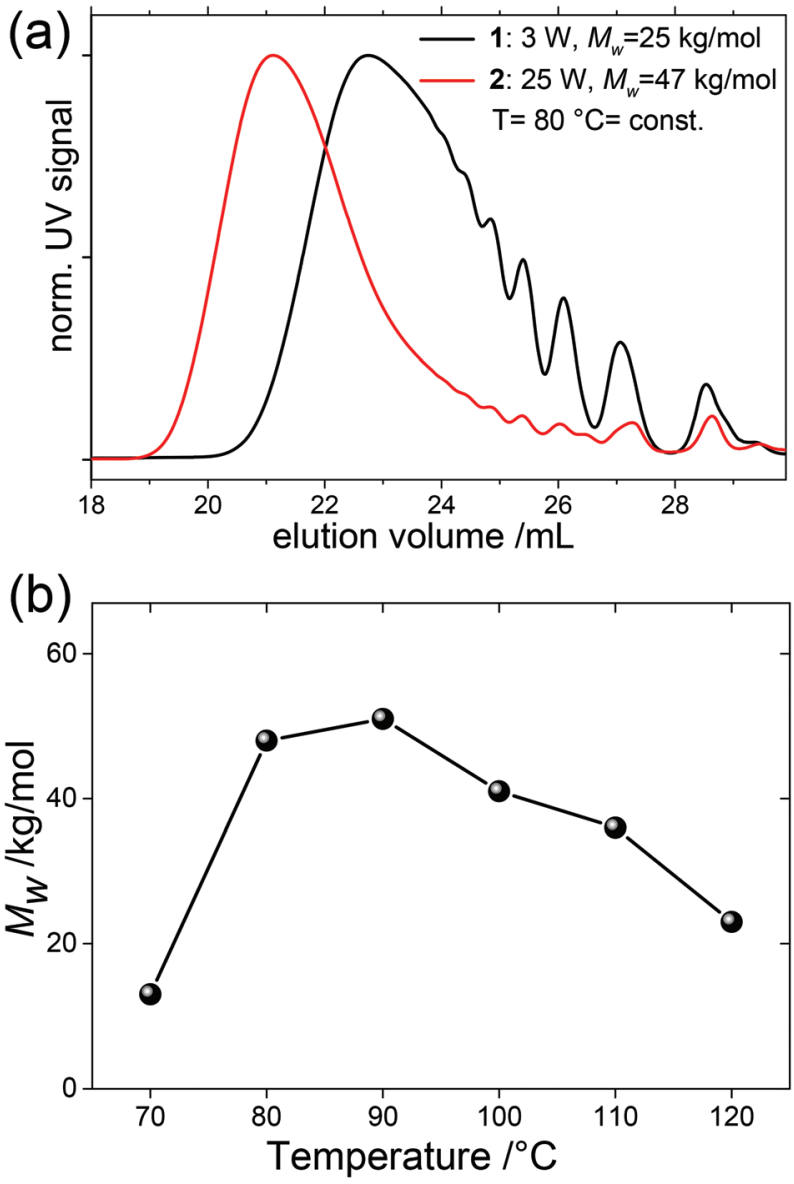

Fig. 2 (a) Effect of microwave irradiation on molecular weight at constant temperature enabled by air cooling. (b) Effect of reaction temperature/microwave power on molecular weight at highest constant cooling.

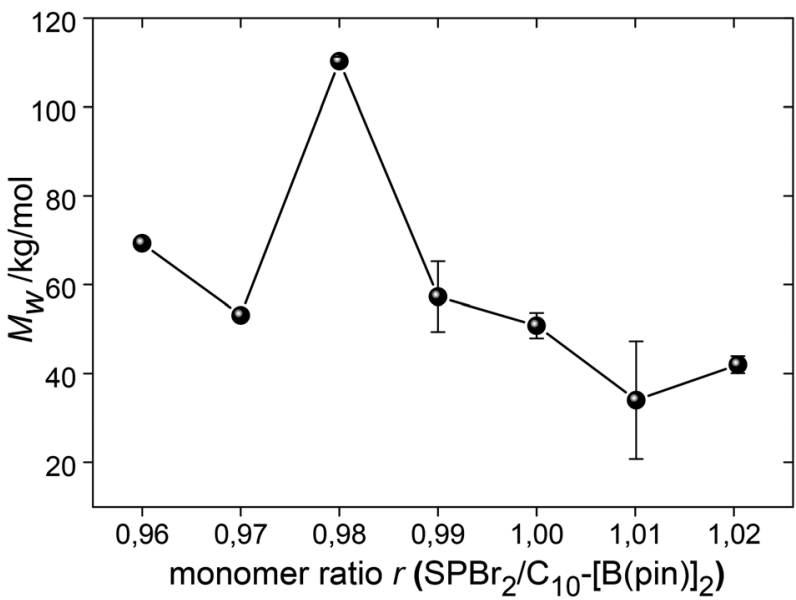

Fig. 3 The dependence of $M_{w}$ on the monomer ratio $r$

tation of all parameters would have led to a very large number of experiments, we did not look into the effects of the phosphine ligand in more detail. In this context, the investigation
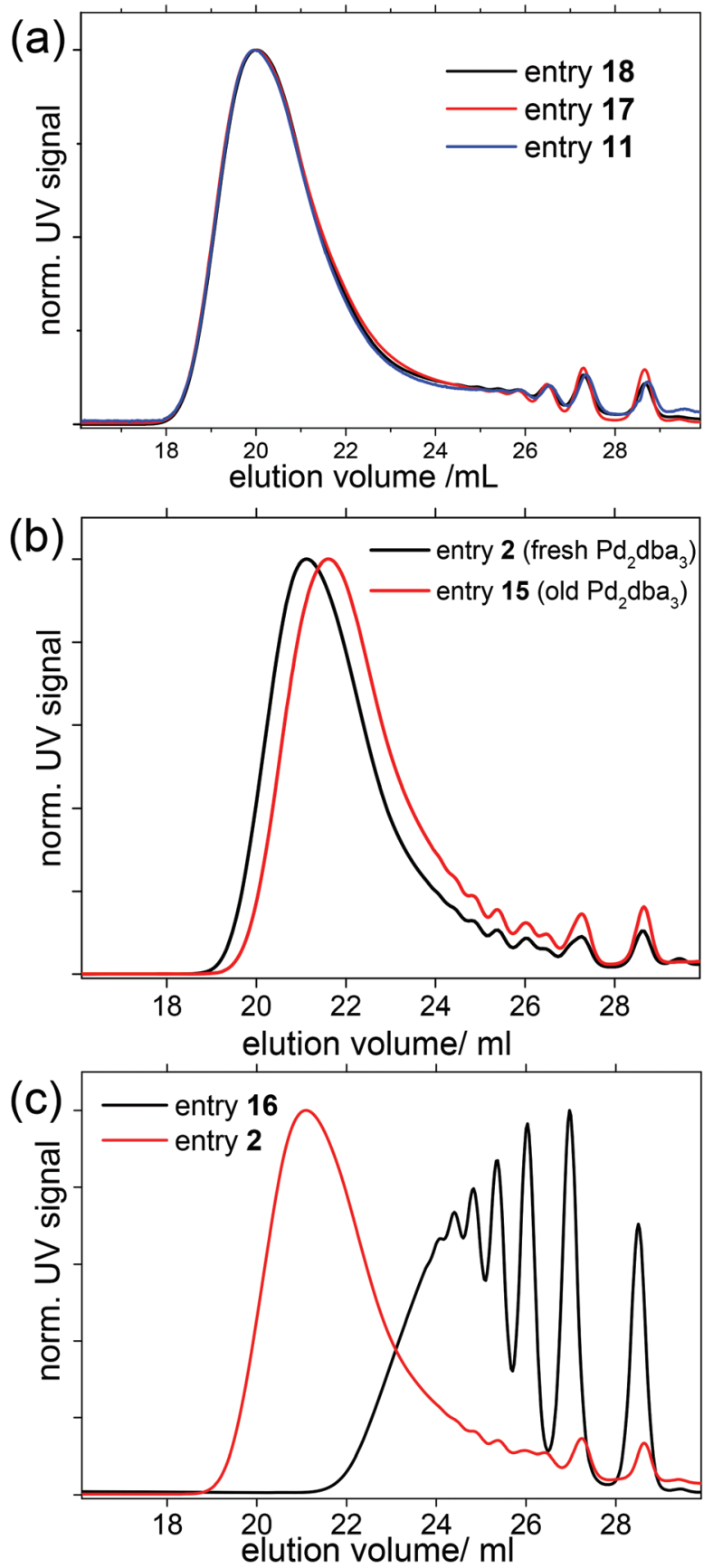

Fig. 4 Reproducibility of the microwave-assisted $\mathrm{SPC}$ of $\mathrm{SPBr}_{2}$ and $\mathrm{C}_{10^{-}}$ $[B(\text { pin })]_{2}$. (a) Three individually polymerized batches were made from freshly prepared, but different stock solutions at different times, resulting in almost the same SEC trace (conditions: $90{ }^{\circ} \mathrm{C}, 2 \mathrm{~h}, 0.98$ equiv. $\mathrm{SPBr}$ ). (b) Effect of aged $\mathrm{Pd}_{2} \mathrm{dba}_{3}$ stock solutions, and (c) effect of aged $\mathrm{C}_{10}-[\mathrm{B}(\mathrm{pin})]_{2}$ stock solutions in which crystallization had reduced the concentration of $\mathrm{C}_{10}-[\mathrm{B}(\mathrm{pin})]_{2}$.

of the ratio of $\mathrm{Pd}_{2} \mathrm{dba}_{3}$ and phosphine appears an interesting parameter to be investigated. ${ }^{67}$ Similar results were obtained upon screening the base. Generally, the role of the base is important here, as the SP units are prone to base-induced cleavage at elevated temperatures. ${ }^{53}$ However, none of the bases 


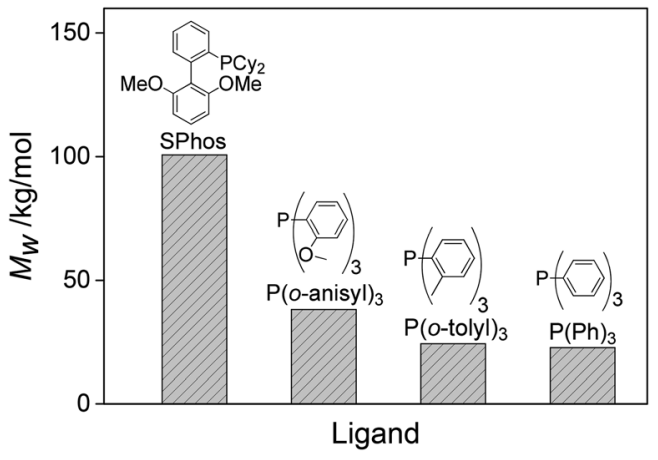

Fig. 5 The influence of the ligand on the $M_{w}$ under identical reaction parameters.

investigated $\left(\mathrm{K}_{3} \mathrm{PO}_{4}, \mathrm{Na}_{2} \mathrm{CO}_{3}, \mathrm{KHCO}_{3}, \mathrm{KF}\right)$ led to higher MWs, and also altering the concentration of aqueous $\mathrm{K}_{2} \mathrm{CO}_{3}$ between $1 \mathrm{M}$ to $3 \mathrm{M}$ did not increase $\mathrm{MW}$.

\section{Mechanistic investigations}

Side and termination reactions. Having defined a set of instrumental and chemical parameters, and a reproducible protocol that led to $\mathrm{P}\left(\mathrm{SP}-\right.$ alt- $\left.\mathrm{C}_{10}\right)$ with $M_{\mathrm{w}} \sim 110 \mathrm{~kg} \mathrm{~mol}^{-1}$ for a reaction time of $2 \mathrm{~h}$, we investigated side and termination reactions that prohibited further increase of molar mass in more detail. As recognized in a previous work, base-induced SP cleavage, leading to salicylaldehyde and 2-methyleneindoline end groups (see Fig. 6a for structures) takes place as an undesired side reaction during polycondensation. ${ }^{53}$ Obviously, this reduces the MW and poses an antagonistic process to the build-up of chains via step-growth. One of the central questions to be pursued here was as to what extent SP cleavage can be suppressed by either changing the base or the reaction temperature. As the use of different bases did not lead to success (not shown), we investigated the effect of temperature on the evolution of MW. For that purpose, samples 3-8 (i.e. temperature-dependent series, see Fig. $2 \mathrm{~b}$ ) were subjected to NMR end group (EG) analysis. Fig. 6 shows a full ${ }^{1} \mathrm{H}$ NMR spectrum of $\mathrm{P}\left(\mathrm{SP}-\right.$ alt $\left.-\mathrm{C}_{10}\right)$ with indicated characteristic chemical shifts of all EGs observed. Depending on conversion and reaction time, bromine, hydrogen, boronic acid pinacol ester, hydroxyl, 2-methyleneindoline, and salicylaldehyde are observed. Not all of them are seen in Fig. 6b, but their chemical shifts are indicated by symbols. For low conversion, typically bromine (on both sides of the asymmetric SP monomer), and boronic acid pinacol ester EGs are observed, while these disappear at higher conversion and are partially replaced by hydrogen as a result of dehalogenation. Protiodeborylation, which is the replacement of $\mathrm{B}(\mathrm{pin}) \mathrm{EGs}$ by hydrogen is not observed, as confirmed by comparison with a model compound $\mathrm{Ph}-\mathrm{O}-\left(\mathrm{CH}_{2}\right)_{6}-\mathrm{O}-\mathrm{Ph}$, where the methylene groups shows up as a triplet at $3.94 \mathrm{ppm}$ (the new aromatic proton is fully covered by backbone signals of $\mathrm{P}\left(\mathrm{SP}-\right.$ alt $\left.-\mathrm{C}_{10}\right){ }^{53}$ Instead, a new triplet is observed at $3.89 \mathrm{ppm}$, which we assigned to $\mathrm{Ph}-\mathrm{OH}$ end caps by a comparison with the spectrum of the model compound 4-heptylphenol (see also Fig. 9a). Such end groups are known to arise as a result of oxidative deborylation. ${ }^{68}$ This side reaction can be used as a marker for the invasion of oxygen into the reaction vessel and hence suggests imprecise experimental conditions. Avoiding this process is especially interesting for conjugated polymers designed for electron transport, as hydroxyl groups are able to trap electrons. ${ }^{69}$

Fig. 7 displays the regions of EGs of interest for several $\mathrm{P}\left(\mathrm{SP}-\right.$ alt $\left.-\mathrm{C}_{10}\right)$ samples polymerized at different temperatures (samples from Fig. 2b). From this figure the occurrence of a maximum in molar mass at $90{ }^{\circ} \mathrm{C}(c f$. Fig. 2) can immediately be understood. At $70^{\circ} \mathrm{C}$, conversion is low as seen by the large amount of boronic acid pinacol ester and bromine EGs, resulting in low MW. At $80{ }^{\circ} \mathrm{C}$, the boronic acid pinacol ester EGs
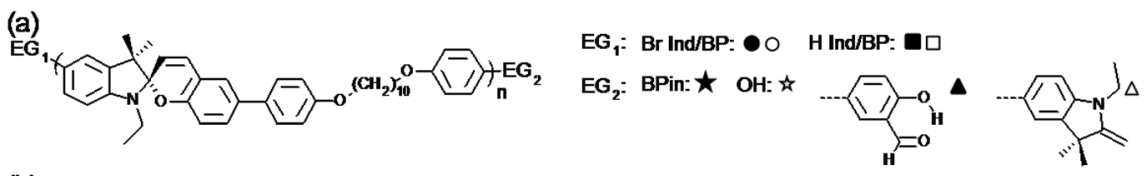

(b)

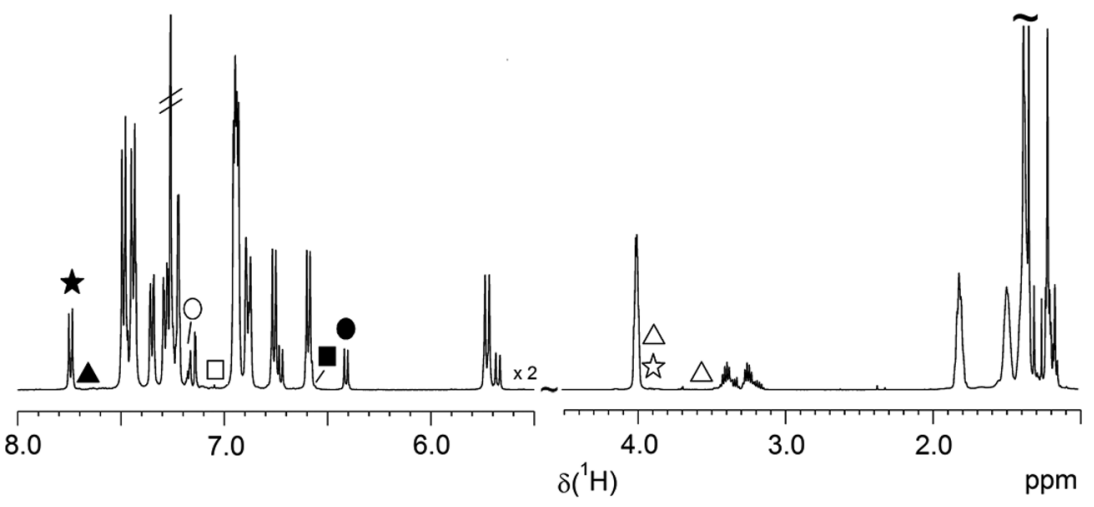

Fig. 6 (a) Chemical structures of observed end groups. (b) Overview of full ${ }^{1} \mathrm{H}$ NMR spectrum of a selected low MW sample of P(SP-alt- $\mathrm{C}_{10}$ ) with indicated chemical shifts of all possible end groups. 

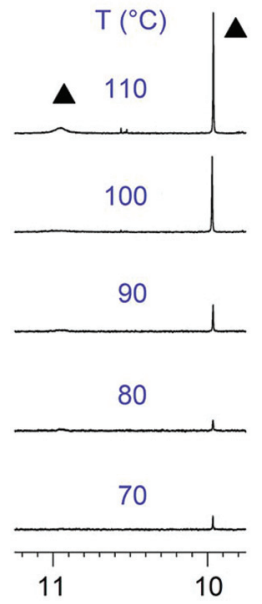
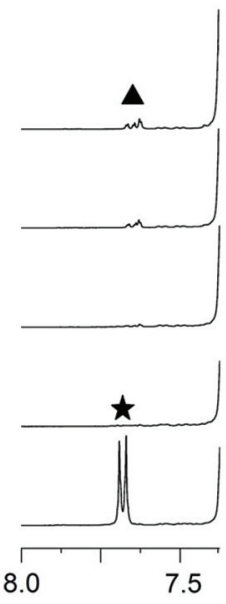
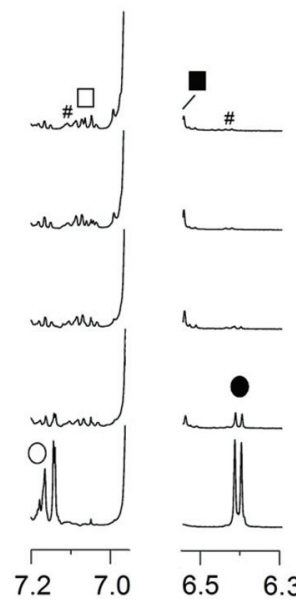

$\delta\left({ }^{1} \mathrm{H}\right)$

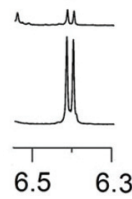

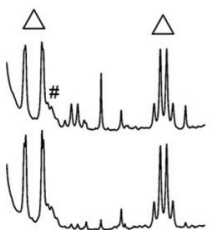
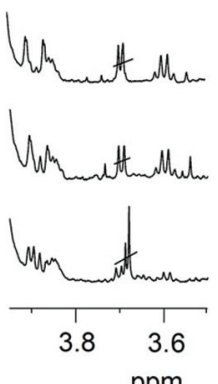

Fig. 7 Regions of relevant end groups of entries 3-8 polymerized at different temperatures for a constant time of $2 \mathrm{~h}$. EG symbols correspond to Fig. 6a. The spectra are normalized to the backbone signal of $\mathrm{OCH}_{2}(3.99 \mathrm{ppm})$. \# marks ${ }^{13} \mathrm{C}$ satellites.

have already vanished completely, while some of the bromine EGs are still visible. At the same time, SP units are being cleaved as can be seen most clearly by the sharp singlet at $\sim 10 \mathrm{ppm}$ (salicylaldehyde end group, the broad and sometimes visible signal at $\sim 11 \mathrm{pm}$ arises from the neighboring $\mathrm{OH}$ group) and the two doublets at 3.9 and $3.6 \mathrm{ppm}$ (methyleneindoline end group). These side reactions are seen already at $70{ }^{\circ} \mathrm{C}$ to a very small extent and become much more pronounced at $110{ }^{\circ} \mathrm{C}$. Consequently, it is the compromise between the highest conversion and lowest degree of SP cleavage that causes the maximum MW seen in Fig. 2b.

From Fig. 7 it is obvious that in order to further improve molar mass the elimination of SP cleavage is most promising. To this end, we subjected prepolymerized $\mathrm{P}\left(\mathrm{SP}-\right.$ alt $\left.^{-\mathrm{C}_{10}}\right)$ to the same microwave reaction conditions as used for polymerization, but in the absence of catalyst, and monitored the cleavage process at $70{ }^{\circ} \mathrm{C}$ and $90{ }^{\circ} \mathrm{C}$ for different times by SEC (Fig. 8a).

At both temperatures, MW decreases with time, but the decrease at $70{ }^{\circ} \mathrm{C}$ is slower. We therefore investigated the evolution of MW during SPC at these two temperatures with time (Table 1, entries 22-29, Fig. 8b). Again, in agreement with the good reproducibility of the SPC protocol, the highest $M_{\mathrm{W}} \sim$ $100 \mathrm{~kg} \mathrm{~mol}^{-1}$ was obtained after $2 \mathrm{~h}$ at $90{ }^{\circ} \mathrm{C}$, and $M_{\mathrm{w}}>80 \mathrm{~kg}$ $\mathrm{mol}^{-1}$ was already obtained after $\sim 30 \mathrm{~min}$. For reaction times longer than $2 \mathrm{~h}$ SP cleavage led to a reduction of the attained chain length. The situation is much different at $70{ }^{\circ} \mathrm{C}$, at which temperature $\sim 3 \mathrm{~h}$ are needed to reach a near-plateau region of $M_{\mathrm{w}}=174 \mathrm{~kg} \mathrm{~mol}^{-1}$, which is now accessible due to suppressed SP cleavage. It can also be anticipated that even higher MWs might be achieved in both cases in the absence of SP cleavage. To extract more mechanistic differences between the series of polymerizations run at 70 and $90{ }^{\circ} \mathrm{C}$, the EGs of these samples were again investigated by NMR spectroscopy. Fig. 9 shows the same regions of interest as a function of
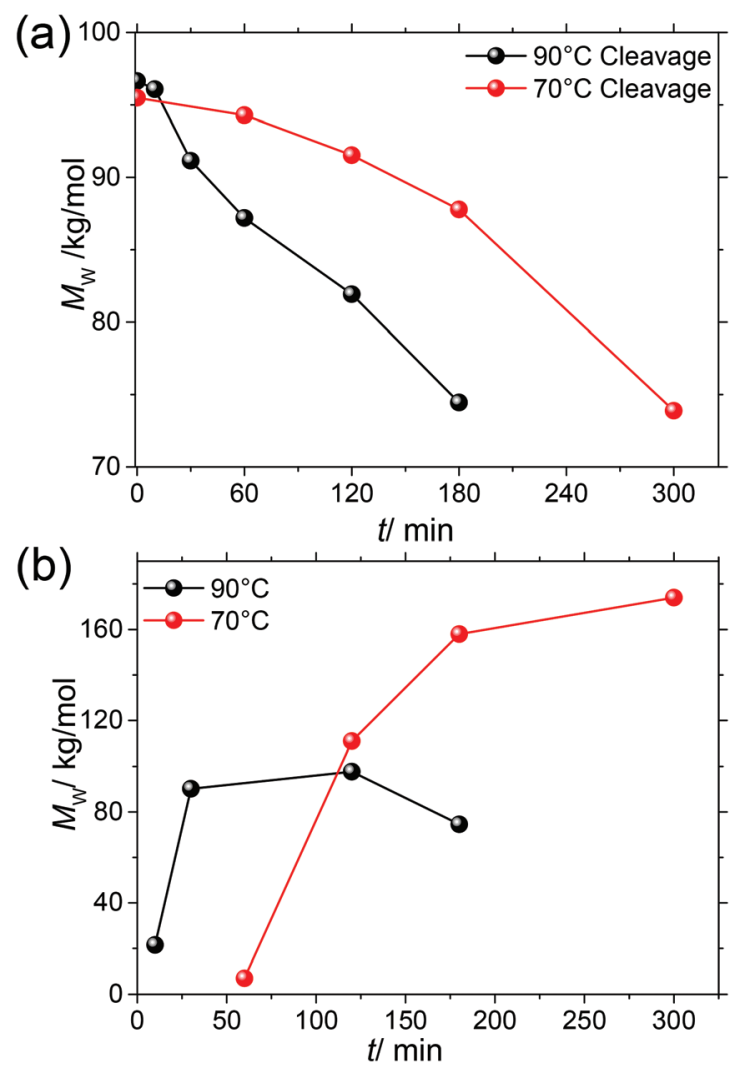

Fig. 8 (a) The SP cleavage process is monitored by SEC for 70 and $90{ }^{\circ} \mathrm{C}$ in the absence of a catalyst. (b) Evolution of $M_{\mathrm{w}}$ with time for 70 and $90{ }^{\circ} \mathrm{C}$ (entries 22-29).

reaction time. It is obvious that next to the reduced SP cleavage at $70{ }^{\circ} \mathrm{C}$, the reaction is much slower at this temperature. While at $90^{\circ} \mathrm{C}$ almost all boronic acid pinacol ester EGs have disappeared after $\sim 30 \mathrm{~min}$, a comparable situation is found at 

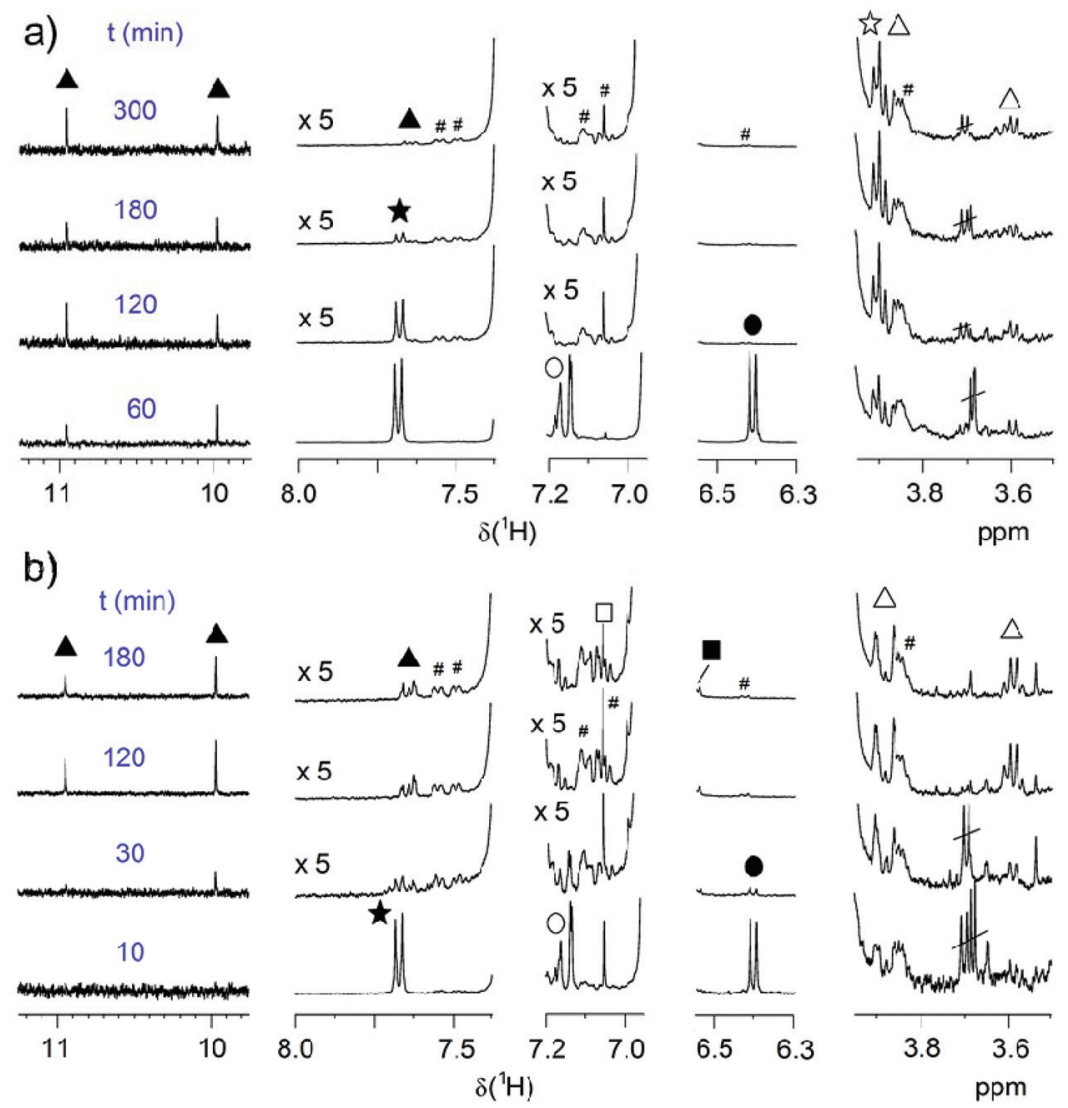

Fig. 9 Time-dependent evolution of EGs of P(SP-alt- $\mathrm{C}_{10}$ ) at a) $70{ }^{\circ} \mathrm{C}$ (entries $26-29$ ) and (b) $90{ }^{\circ} \mathrm{C}$ (entries 22-25). EG symbols correspond to Fig. 6a. The spectra are normalized to the backbone signal of $\mathrm{OCH}_{2}(3.99 \mathrm{ppm})$. \# marks ${ }^{13} \mathrm{C}$ satellites.

$70{ }^{\circ} \mathrm{C}$ after $\sim 180 \mathrm{~min}$. However, another difference is an increased degree of debromination at $90{ }^{\circ} \mathrm{C}$, seen by the $\mathrm{SP}-\mathrm{H}$ EGs ( $c f$. Fig. 6). The absence of this side reaction at $70^{\circ} \mathrm{C}$ might additionally contribute to the higher MW plateau that can be reached at reduced temperature. Dehalogenation is commonly observed in basically all Pd-catalyzed polycondensations such as SPC, direct arylation polycondensation, or Stille polycondensation. ${ }^{21,53,70-74}$ What is observed at $70{ }^{\circ} \mathrm{C}$, however, is the presence of phenolic end groups demonstrated by the characteristic triplet at $3.89 \mathrm{ppm}$ (comparison with 4-heptylphenol as model compound) as a result of oxidative deborylation. ${ }^{68}$ This side reaction is usually accompanied by boronic acid ester homocoupling, detection of which is not possible here. Thus, hydroxylated EGs can be used as markers to identify SPCs in which invasion of oxygen could not be impeded. ${ }^{21}$ As this characteristic triplet is already present from the beginning of the polymerization at $70{ }^{\circ} \mathrm{C}$, we can exclude the possibility of oxygen contamination during prolonged reaction times and hence conclude that oxygen must have been present from the beginning due to incomplete degassing. We also note that the occurrence of this side reaction at $70{ }^{\circ} \mathrm{C}$ is only apparently coincidental, as the slower kinetics of the reaction at $70{ }^{\circ} \mathrm{C}$ leave much more time for other reactions such as oxidative deborylation.
As the experimental parameters applied here were always the same, we assumed that residual oxygen had also been present in all other polymerization entries, but was simply not detected due to the fast consumption of $\mathrm{C}_{10^{-}}[\mathrm{B}(\mathrm{pin})]_{2}$ at $90{ }^{\circ} \mathrm{C}$. We also note that the small degree of hydroxyl-end capped chains does not seem to affect molar mass negatively. Thus, these detailed NMR end group analyses not only allow optimizing SPC reactions through the identification and elimination of side reactions, but also enable a detailed understanding of the factors that limit conversion to approach unity.

\section{Ring molecules}

An extension of the Carothers equation includes the possibility of cyclic molecules at any stage of a step-growth polymerization. ${ }^{34}$ Since the formation of ring molecules does not alter the ratio of functional groups, it does not limit maximum conversion. This is not the case for the molecular weight distribution. If a significant amount of cyclic molecules is formed, molar mass is lower, dispersity can be higher, and the number average molecular weights determined by NMR end group analysis are overestimated. In $\mathrm{P}\left(\mathrm{SP}-\right.$ alt- $\left.\mathrm{C}_{10}\right)$, the flexible alkyl chain of the comonomer $\mathrm{C} 10-[\mathrm{B}(\mathrm{pin})]_{2}$ should permit ring formation. However, differently than for strained fully aromatic rings, the chemical shifts of the flexible rings formed here are unlikely to 

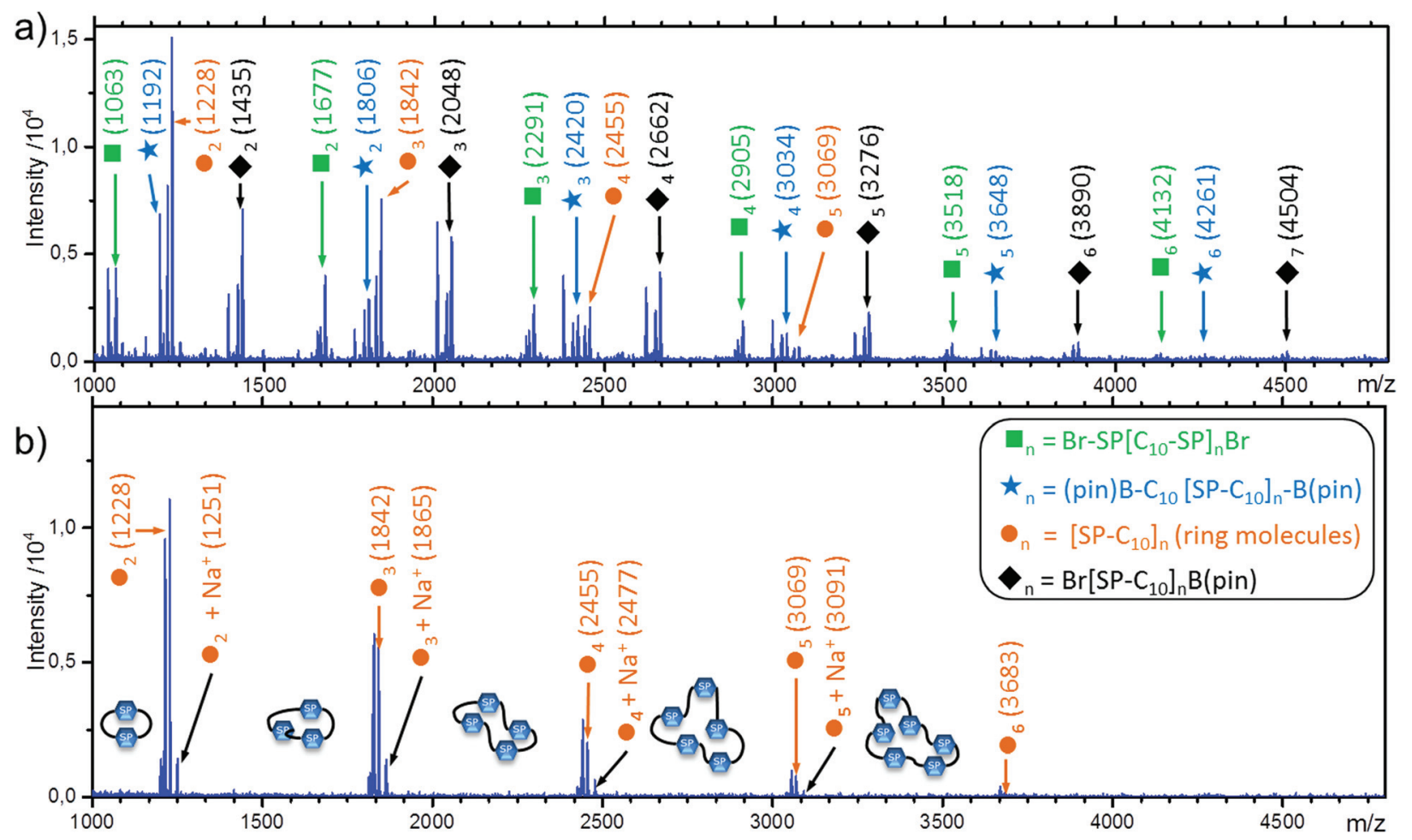

Fig. 10 MALDI-TOF analysis of unfractionated polymers with a) $M_{\mathrm{w}}=21.5 \mathrm{~kg} \mathrm{~mol}^{-1}$ and b) $M_{\mathrm{w}}=174 \mathrm{~kg} \mathrm{~mol}^{-1}$.

differ much from the linear analogs. ${ }^{17}$ In a $\mathrm{P}\left(\mathrm{SP}-\right.$ alt $\left.-\mathrm{C}_{10}\right)$ sample with low molar mass $\left(M_{\mathrm{w}}=21.9 \mathrm{~kg} \mathrm{~mol}^{-1}\right.$, entry 22), different series of oligomers were detected via MALDI-TOF mass spectrometry. Besides the expected series of oligomers with all combinations of $\mathrm{Br}$ - and/or Bpin-EGs, ring molecules with sizes $n=2-6$ were clearly identified (Fig. 10a). The presence of all these oligomers shows that the SPC reaction takes place as expected, and the presence of ring molecules is in line with the flexibility of the comonomer $\mathrm{C}_{10}-[\mathrm{B}(\mathrm{pin})]_{2}{ }^{75}$ The MALDI-TOF mass spectrum of an unfractionated high molar mass sample $\left(M_{\mathrm{w}}=174 \mathrm{~kg} \mathrm{~mol}^{-1}\right.$, entry 29), only exhibited the peak series of the ring molecules (Fig. 10b), as all other chains were too large to be detected. Thus, we can assume that larger rings are present as well, but these are simply invisible by both NMR spectroscopy and MALDI-TOF. The occurrence of cyclic molecules can also explain the several signals of the SEC curves at large elution volume (see Fig. 4).

\section{Mechanically-induced SP $\rightarrow$ MC isomerization}

The typical experiment for verifying the mechanochromic response of a polymeric material is a stress-strain test. ${ }^{44-50}$ However, the high $T_{\mathrm{g}}$ of $\mathrm{P}\left(\mathrm{SP}-\right.$ alt- $\left.\mathrm{C}_{10}\right)$ of $129{ }^{\circ} \mathrm{C}$ and its nonductile character did not allow for such experiments, as films ruptured before developing color. Plasticizing the material or stress-strain under elevated temperature can circumvent this behavior, which was not attempted here. ${ }^{76}$ The light-induced $\mathrm{SP} \rightarrow \mathrm{MC}$ isomerization did not occur within the time scales investigated (not shown), most likely due to the high $T_{\mathrm{g}}$ of $\mathrm{P}\left(\mathrm{SP}-\right.$ alt $\left.-\mathrm{C}_{10}\right) \cdot{ }^{77}$ Instead, the mechanochromic response was assessed by embossing films of $\mathrm{P}\left(\mathrm{SP}-\right.$ alt $\left.-\mathrm{C}_{10}\right)$ with a metal stamp (Fig. 11). These conditions led to the development of a green color at the embossed regions, which we found to be metastable. Embossed films kept their color for about 2-3 d at room temperature before undergoing a back reaction to the SP form. Under solvent vapor annealing with good solvents such as THF, the films lost color within about minutes, but in nonsolvents such as hexanes or methanol this effect was absent. Interestingly, thermal annealing at temperatures $>60{ }^{\circ} \mathrm{C}$ discolored embossed films instantaneously. From these experiments, it is evident that the back reaction is facilitated with increasing mobility within the film, which is either induced by solvent vapor or thermal annealing.

An interesting question is as to why a green color is observed, which is unusual compared to known SP derivatives. ${ }^{1}$ We assume that this color is caused by two factors, (i) phenyl substitution para to the phenolate part of the MC form, and (ii) due to aggregation effects arising from the high SP density in the film. However, aggregation effects are more complex as a result of the different types of alignment of the dipole moments. This will be looked at in detail in future investigations. Meanwhile, a comparison of this behaviour with several reported SP polymer architectures is not straightforward, as several parameters are different. First, in most cases a common SP derivative carrying a nitro group in 6-position is used, while a phenyl ring was employed 
a)

as prepared

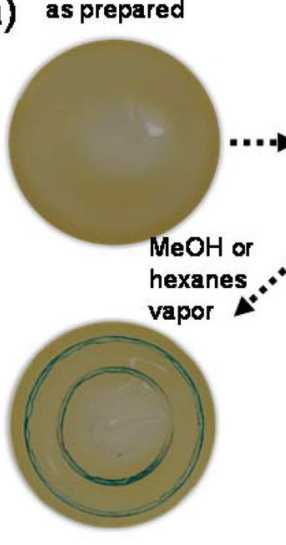

embossed

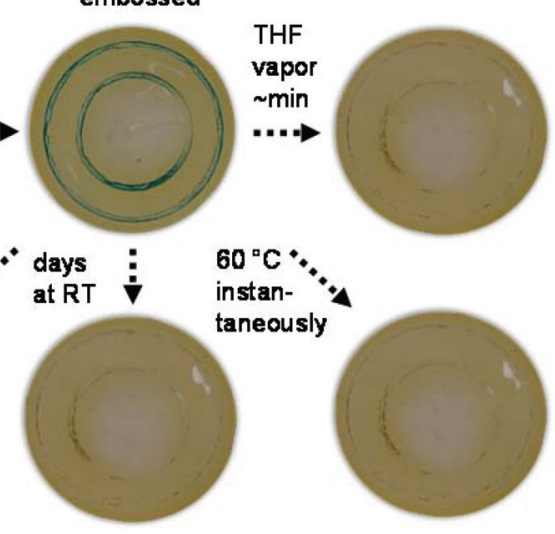

b)

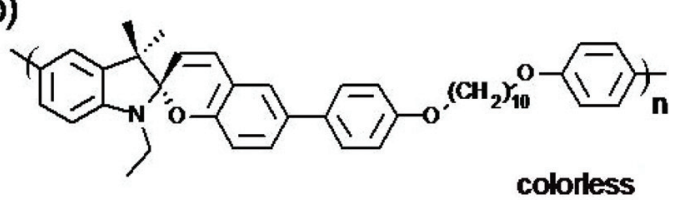

colorless

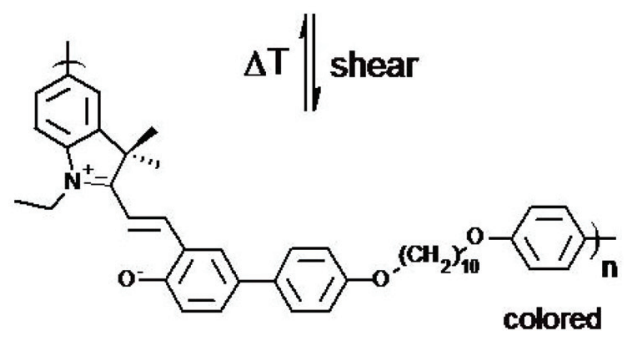

Fig. 11 Mechanochromic behavior of $\mathrm{P}\left(\mathrm{SP}-\right.$ alt $-\mathrm{C}_{10}$ ) probed by embossing. (a) Mechanochromic response and back reaction to SP in the solid state and (b) reversible isomerization from the colorless SP form to the colored MC form induced by the shear forces of the metal stamp.

here. ${ }^{44,45,47-50,76}$ Second, the position of polymer chain linkage is different as well, which is likely to be important for the mechanochromic response, although this detail has not yet been investigated consistently. ${ }^{44}$ Third, the mentioned difference of the glass transition temperature strongly influences mechanochromic activation. ${ }^{76}$ Clarifying whether the external force is transduced to the SP unit by putting strain on the polymer chains or simply transferred through the glassy and stiff nature of $\mathrm{P}\left(\mathrm{SP}-\right.$ alt- $\left.\mathrm{C}_{10}\right)$ is not trivial and not attempted here. To this end, the potential mechanochromic behavior of low molar mass SP derivatives that are not covalently linked to a polymer chain but rather dispersed in a glassy polymer matrix of varying $T_{\mathrm{g}}$ might help, which will be addressed by future experiments. The possible reasons of the thermally facilitated back reaction appeared to be most interesting. To obtain a deeper understanding, the energetics and the nature of the transition barrier of the $\mathrm{SP} \rightarrow \mathrm{MC}$ reaction of the model compound SP-Ph-OMe were elucidated in the gas phase via density functional theory (DFT, Fig. 12). ${ }^{53}$ This model compound exhibits the same substitution pattern on the benzopyran side of $\mathrm{P}\left(\mathrm{SP}-\right.$ alt- $\left.\mathrm{C}_{10}\right)$ and thus represents the relevant $\mathrm{SP}$ chromophore. Importantly, we did not model the force-activated SP $\rightarrow$ MC isomerization directly, but rather looked at the energetics of the SP and MC forms as well as the height of the barrier between the two states. The investigation of the barrier is especially interesting for the discussion of the thermally accelerated $\mathrm{MC} \rightarrow \mathrm{SP}$ back reaction as discussed below.

The energetics of the transition path obtained from DFT are displayed in Fig. 12a, where the path length is defined as the geometrically added distances between all atomic positions (corresponding to a mechanical experiment). The energies of SP and MC isomers differ by $0.02 \mathrm{eV}$ (the lowest energy

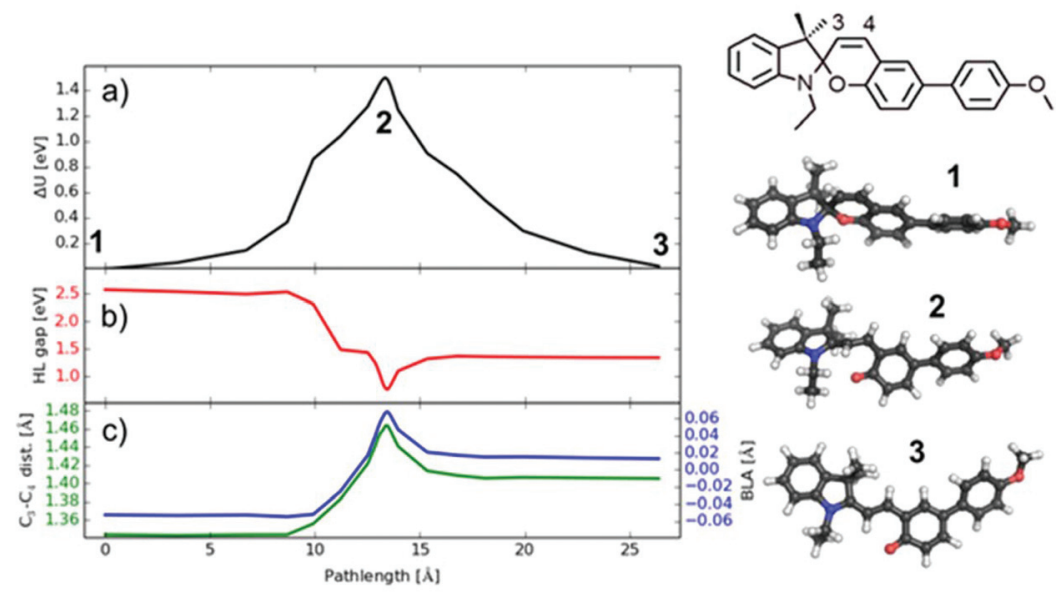

Fig. 12 DFT calculations of the mechanically-induced SP $\rightarrow M C$ isomerization of the model compound SP-PhOMe. (a) Reaction path for the transition from SP-PhOMe 1 via the transition state 2 to the lowest MC isomer 3 (TTC). (b) The corresponding HOMO-LUMO (H-L) gap along the same path length, $1 \mathrm{eV}=23.06 \mathrm{kcal} \mathrm{mol}^{-1}=96.49 \mathrm{~kJ} \mathrm{~mol}^{-1}$. (c) The $\mathrm{C}_{3}-\mathrm{C}_{4}$ bond length and the value of bond length alternation (BLA). The chemical structure of the model compound and the calculated geometries of 1, 2 and 3 are depicted, C: black, N: blue, O: red, H: white. 
TTC isomer is investigated here, which is slightly lower than the CTT isomer treated by Komber et $a l .{ }^{53}$ ), but are separated by a high barrier of $1.54 \mathrm{eV}$ in agreement with barrier heights found in other spiropyrans. ${ }^{41,78}$ The barrier consists of two contributions: a first shoulder at $10 \AA$ A path length followed by the highest energy around $14 \AA$ path length. Interestingly, the $\mathrm{C}-\mathrm{O}$ bond breaks at the position of the first shoulder, as seen by the sharp drop in the energy gap between the highest occupied (HOMO) and lowest unoccupied molecular orbital (LUMO) (Fig. 12b), showing a color change when MC is formed. The successive barrier at $14 \AA$ path length arises from an internal rotation of the two halves of the molecule and affects the color less. The additional dip in the HOMO/LUMO gap in this region is related to the necessary bond reorganization that can be characterized by the bond length alternation (BLA) value $\mathrm{e}^{78}$ or equivalently by the length of the $\mathrm{C}_{3}-\mathrm{C}_{4}$ bond (see Fig. 12). This bond has a double bonding character in SP (as compared to the CC bond length of $1.33 \AA$ in ethylene) and exhibits a mixed character in MC (the single bond in ethane has $1.52 \AA)$. Exactly in the region of the barrier this bond takes a more single character which is energetically unfavorable.

The energy difference between SP and MC is very small and the isomers are separated by a large barrier. This suggests very little probability for a back transition to SP once MC is formed under the experimental conditions (thermal energy is $0.03 \mathrm{eV}$ at $60{ }^{\circ} \mathrm{C}$ ), and decoloration via temperature observed here has to be caused by other effects. One possibility could be that the rather stiff polymer matrix does not allow the MC form to find its most stable TTC form. In other words, a possible restriction of the internal rotation would leave the MC form in a final state of higher energy, from which a thermally-induced back reaction could be possible even at low temperatures. An alternative explanation is that energy is stored in the deformed polymer matrix during the embossing process which can be released very fast at $60{ }^{\circ} \mathrm{C}$, and which is sufficient to overcome the barrier between MC and SP. If so, this decoloration process might pose a possibility to directly visualize relaxation processes in polymeric materials.

\section{Conclusions}

We have presented an optimized microwave-assisted protocol towards the reproducible synthesis of a high molecular weight mechanochromic copolymer on a small scale by SuzukiMiyaura polycondensation. Using this protocol, small amounts of monomer can be copolymerized with good molecular weight reproducibility, which enables the screening of a large number of experimental parameters for a limited amount of monomer available. The herein screened parameters included stoichiometry, base, ligand, time, temperature, and power. The process of optimization was guided by NMR end group analysis, which revealed base-induced cleavage of spiropyran (SP), dehalogenation, and oxidative deborylation as side reactions. Lower temperatures decreased the extent of SP cleavage and dehalogenation, which enabled higher molar masses of $\mathrm{P}(\mathrm{SP}-$ alt- $\mathrm{C}_{10}$ ) up to $M_{\mathrm{w}}=174 \mathrm{~kg} \mathrm{~mol}^{-1}$ to be obtained for longer reaction times. The copolymer exhibits an interesting mechanochromic behavior. Embossed films are stained green at regions of high shear forces and decolorize fast at temperatures wellbelow the glass transition temperature, despite a relatively high barrier of $1.54 \mathrm{eV}$. These results might be an indication that energy can be stored in the polymer matrix by embossing, release of which can be visualized by the $\mathrm{MC} \rightarrow$ SP back reaction. Possible task fields of such SP copolymers with a high glass transition temperature involve applications in which high shear fields and/or relaxation processes in polymeric materials are to be visualized by reversible color changes.

\section{Author contributions}

The manuscript was written through contributions of all authors. All authors have given approval to the final version of the manuscript.

\section{Funding sources}

The Fonds der Chemischen Industrie (FCI) and the Innovationsfond Forschung of the Universität Freiburg are greatly acknowledged for funding.

\section{Acknowledgements}

The authors thank M. Hagios for GPC measurements. Computational resources from FZ Jülich are gratefully acknowledged.

\section{References}

1 M. Rehahn, A. D. Schlüter, G. Wegner and W. J. Feast, Polymer, 1989, 1060-1062.

2 A. D. Schlüter, J. Polym. Sci., 2001, 39, 1533-1556.

3 J. Sakamoto, M. Rehahn, G. Wegner and A. D. Schlüter, Macromol. Rapid Commun., 2009, 30, 653-687.

4 R. Kandre, K. Feldman, H. E. H. Meijer, P. Smith and A. D. Schlüter, Angew. Chem., Int. Ed., 2007, 46, 4956-4959.

5 Y. Xu, S. Jin, H. Xu, A. Nagai and D. Jiang, Chem. Soc. Rev., 2013, 42, 8012-8031.

6 F. Vilela, K. Zhang and M. Antonietti, Energy Environ. Sci., 2012, 5, 7819-7832.

7 F. Wang, J. Mielby, F. H. Richter, G. Wang, G. Prieto, T. Kasama, C. Weidenthaler, H.-J. Bongard, S. Kegnæs, A. Fürstner and F. Schüth, Angew. Chem., Int. Ed., 2014, 53, 8645-8648.

8 A. C. Grimsdale, K. Leok Chan, R. E. Martin, P. G. Jokisz and A. B. Holmes, Chem. Rev., 2009, 109, 897-1091.

9 P. M. Beaujuge and J. R. Reynolds, Chem. Rev., 2010, 110, 268-320. 
10 D. T. McQuade, A. E. Pullen and T. M. Swager, Chem. Rev., 2000, 100, 2537-2574.

11 Y.-J. Cheng, S.-H. Yang and C.-S. Hsu, Chem. Rev., 2009, 109, 5868-5923.

12 F. E. Goodson, T. I. Wallow and B. M. Novak, Macromolecules, 1998, 31, 2047-2056.

13 M. Jayakannan, J. L. J. van Dongen and R. A. J. Janssen, Macromolecules, 2001, 34, 5386-5393.

14 Z. Bo and A. D. Schlüter, Chem. - Eur. J., 2000, 6, 32353241.

15 J. Murage, J. W. Eddy, J. R. Zimbalist, T. B. McIntyre, Z. R. Wagner and F. E. Goodson, Macromolecules, 2008, 41, 7330-7338.

16 M. Liu, Y. Chen, C. Zhang, C. Li, W. Li and Z. Bo, Polym. Chem., 2013, 4, 895-899.

17 B. Hohl, L. Bertschi, X. Zhang, A. D. Schlüter and J. Sakamoto, Macromolecules, 2012, 45, 5418-5426.

18 M. Jayakannan, X. Lou, J. L. J. van Dongen and R. A. J. Janssen, J. Polym. Sci., Part A: Polym. Chem., 2005, 43, 1454-1462.

19 Y. Kim, S. Cook, J. Kirkpatrick, J. Nelson, J. R. Durrant, D. D. C. Bradley, M. Giles, M. Heeney, R. Hamilton and I. McCulloch, J. Phys. Chem. C, 2007, 111, 81378141.

20 J. K. Park, J. Jo, J. H. Seo, J. S. Moon, Y. D. Park, K. Lee, A. J. Heeger and G. C. Bazan, Adv. Mater., 2011, 23, 24302435.

21 M. Sommer, H. Komber, S. Huettner, R. Mulherin, P. Kohn, N. C. Greenham and W. T. S. Huck, Macromolecules, 2012, 45, 4142-4151.

22 H. Komber, V. Senkovskyy, R. Tkachov, K. Johnson, A. Kiriy, W. T. S. Huck and M. Sommer, Macromolecules, 2011, 44, 9164-9172.

23 R. J. Kline, M. D. McGehee, E. N. Kadnikova, J. Liu and J. M. J. Fréchet, Adv. Mater., 2003, 15, 1519-1522.

24 A. Gasperini and K. Sivula, Macromolecules, 2013, 46, 93499358.

25 F. Wiesbrock, R. Hoogenboom, M. Leenen, S. F. G. M. van Nispen, M. van der Loop, C. H. Abeln, A. M. J. van den Berg and U. S. Schubert, Macromolecules, 2005, 38, 79577966.

26 K. Kempe, C. R. Becer and U. S. Schubert, Macromolecules, 2011, 44, 5825-5842.

27 C. O. Kappe, Angew. Chem., Int. Ed., 2004, 116, 6408-6443.

28 I. R. Baxendale, C. M. Griffiths-Jones, S. V. Ley and G. K. Tranmer, Chem. Weinh. Bergstr. Ger., 2006, 12, 44074416.

29 M. Larhed and A. Hallberg, J. Org. Chem., 1996, 61, 95829584.

30 P. Lindström, J. Tierney and J. Westman, Tetrahedron, 2001, 57, 9225-9283.

31 L. André, Microwaves in Organic Synthesis, WILEY-VCH Verlag, Weinheim, 1. Auflage., 2002.

32 N. E. Leadbeater and M. Marco, J. Org. Chem., 2003, 68, 888-892.

33 W. H. Carothers, J. Am. Chem. Soc., 1929, 51, 2548-2559.
34 C. Wutz and H. R. Kricheldorf, Macromol. Theory Simul., 2012, 21, 266-271.

35 J. Sakamoto, M. Rehahn and A. D. Schlüter, Design and Synthesis of Conjugated Polymers, WILEY-VCH, Weinheim, 2010.

36 B. S. Nehls, S. Füldner, E. Preis, T. Farrell and U. Scherf, Macromolecules, 2005, 38, 687-694.

37 F. Galbrecht, T. W. Bünnagel, T. Farrell and U. Scherf, Macromol. Rapid Commun., 2007, 28, 387-394.

38 S. Schmücker and D. Kuckling, Macromol. Chem. Phys., 2012, 213, 1725-1734.

39 A. Britze, J. Jacob, V. Choudhary, V. Moellmann, G. Grundmeier, H. Luftmann and D. Kuckling, Polymer, 2010, 51, 5294-5303.

40 W. Zhang, P. Lu, Z. Wang and Y. Ma, J. Polym. Sci., Part A: Polym. Chem., 2013, 51, 1950-1955.

41 V. I. Minkin, Chem. Rev., 2004, 104, 2751-2776.

42 R. Klajn, Chem. Soc. Rev., 2014, 43, 148-184.

43 S. L. Potisek, D. A. Davis, N. R. Sottos, S. R. White and J. S. Moore, J. Am. Chem. Soc., 2007, 129, 1380813809.

44 D. A. Davis, A. Hamilton, J. Yang, L. D. Cremar, D. Van Gough, S. L. Potisek, M. T. Ong, P. V. Braun, T. J. Martínez, S. R. White, J. S. Moore and N. R. Sottos, Nature, 2009, 459, 68-72.

45 G. O'Bryan, B. M. Wong and J. R. McElhanon, ACS Appl. Mater. Interfaces, 2010, 2, 1594-1600.

46 K. M. Wiggins, J. N. Brantley and C. W. Bielawski, Chem. Soc. Rev., 2013, 42, 7130-7147.

47 G. R. Gossweiler, G. B. Hewage, G. Soriano, Q. Wang, G. W. Welshofer, X. Zhao and S. L. Craig, ACS Macro Lett., 2014, 3, 216-219.

48 C. K. Lee, D. A. Davis, S. R. White, J. S. Moore, N. R. Sottos and P. V. Braun, J. Am. Chem. Soc., 2010, 132, 1610716111.

49 C. K. Lee, C. E. Diesendruck, E. Lu, A. N. Pickett, P. A. May, J. S. Moore and P. V. Braun, Macromolecules, 2014, 47, 2690-2694.

50 S. Jiang, L. Zhang, T. Xie, Y. Lin, H. Zhang, Y. Xu, W. Weng and L. Dai, ACS Macro Lett., 2013, 2, 705-709.

51 Y. Chen and R. P. Sijbesma, Macromolecules, 2014, 47, 3797-3805.

52 M. Sommer and H. Komber, Macromol. Rapid Commun., 2013, 34, 57-62.

53 H. Komber, S. Müllers, F. Lombeck, A. Held, M. Walter and M. Sommer, Polym. Chem., 2014, 5, 443-453.

54 V. A. Krongauz and E. S. Goldburt, Macromolecules, 1981, 14, 1382-1386.

55 X. Guo, Y. Zhou, D. Zhang, B. Yin, Z. Liu, C. Liu, Z. Lu, Y. Huang and D. Zhu, J. Org. Chem., 2004, 69, 89248931.

56 G. Copley, J. G. Gillmore, J. Crisman, G. Kodis, C. L. Gray, B. R. Cherry, B. D. Sherman, P. A. Liddell, M. M. Paquette, L. Kelbauskas, N. L. Frank, A. L. Moore, T. A. Moore and D. Gust, J. Am. Chem. Soc., 2014, 136, 11994-12003. 
57 G. M. Sheldrick, Acta Crystallogr., Sect. A: Fundam. Crystallogr., 2008, 64, 112-122.

58 H. Putz and K. Brandenburg, Diamond: Crystal and Molecular Structure Visualization, vol. 3.2i, Crystal Impact GbR, Bonn, Germany, 2012.

59 J. P. Perdew, K. Burke and M. Ernzerhof, Phys. Rev. Lett., 1996, 77, 3865-3868.

60 P. E. Blöchl, Phys. Rev. B: Condens. Matter, 1994, 50, 1795317979.

61 J. J. Mortensen, L. B. Hansen and K. W. Jacobsen, Phys. Rev. B: Condens. Matter, 2005, 71, 035109.

62 J. Enkovaara, C. Rostgaard, J. J. Mortensen, J. Chen, M. Dułak, L. Ferrighi, J. Gavnholt, C. Glinsvad, V. Haikola, H. A. Hansen, H. H. Kristoffersen, M. Kuisma, A. H. Larsen, L. Lehtovaara, M. Ljungberg, O. Lopez-Acevedo, P. G. Moses, J. Ojanen, T. Olsen, V. Petzold, N. A. Romero, J. StausholmMøller, M. Strange, G. A. Tritsaris, M. Vanin, M. Walter, B. Hammer, H. Häkkinen, G. K. H. Madsen, R. M. Nieminen, J. K. Nørskov, M. Puska, T. T. Rantala, J. Schiøtz, K. S. Thygesen and K. W. Jacobsen, J. Phys. Condens. Matter, 2010, 22, 253202.

63 G. Henkelman, B. P. Uberuaga and H. Jónsson, J. Chem. Phys., 2000, 113, 9901-9904.

64 J. M. G. Cowie, Chemie und Physik der synthetischen Polymere, Friedr. Vieweg \& Sohn Verlagsgesellschaft $\mathrm{mbH}$, Wiesbaden, 1997.

65 W. H. Carothers, Trans. Faraday Soc., 1936, 32, 39-49.

66 R. Martin and S. L. Buchwald, Acc. Chem. Res., 2008, 41, 1461-1473.
67 C. Amatore and A. Jutand, Coord. Chem. Rev., 1998, 178180, Part 1, 511-528.

68 C. Adamo, C. Amatore, I. Ciofini, A. Jutand and H. Lakmini, J. Am. Chem. Soc., 2006, 128, 6829-6836.

69 L.-L. Chua, J. Zaumseil, J.-F. Chang, E. C.-W. Ou, P. K.H. Ho, H. Sirringhaus and R. H. Friend, Nature, 2005, 434, 194-199.

70 F. Lombeck, R. Matsidik, H. Komber and M. Sommer, Macromol. Rapid Commun., 2015, 36, 231-237.

71 F. Lombeck, H. Komber, S. I. Gorelsky and M. Sommer, ACS Macro Lett., 2014, 819-823.

72 A. Luzio, D. Fazzi, F. Nübling, R. Matsidik, A. Straub, H. Komber, E. Giussani, S. E. Watkins, M. Barbatti, W. Thiel, E. H. Gann, L. Thomsen, C. R. McNeill, M. Caironi and M. Sommer, Chem. Mater., 2014, 26, 6233-6240.

73 M. Brinkmann, E. Gonthier, S. Bogen, K. Tremel, S. Ludwigs, M. Hufnagel and M. Sommer, ACS Nano, 2012, 6, 10319-10326.

74 O. Navarro, N. Marion, Y. Oonishi, R. A. Kelly and S. P. Nolan, J. Org. Chem., 2006, 71, 685-692.

75 Y. Sangvikar, K. Fischer, M. Schmidt, A. D. Schlüter and J. Sakamoto, Org. Lett., 2009, 11, 4112-4115.

76 B. A. Beiermann, D. A. Davis, S. L. B. Kramer, J. S. Moore, N. R. Sottos and S. R. White, J. Mater. Chem., 2011, 21, 8443-8447.

77 G. K. Such, R. A. Evans and T. P. Davis, Macromolecules, 2006, 39, 1391-1396.

78 Y. Sheng, J. Leszczynski, A. A. Garcia, R. Rosario, D. Gust and J. Springer, J. Phys. Chem. B, 2004, 108, 16233-16243. 